\title{
DYNAMIC RESPONSE OF A ROTATING BEAM SUBJECTED TO AN ACCELERATING DISTRIBUTED SURFACE FORCE
}

\author{
A. Argento \\ Department of Mechanical Engineering, The University of Michigan-Dearborn, \\ Dearborn, Michigan 48128-1491, U.S.A. \\ AND \\ R. A. SCOTT \\ Department of Mechanical Engineering and Applied Mechanics, University of Michigan, \\ Ann Arbor, Michigan 48109-2125, U.S.A.
}

(Received 5 October 1990, and in final form 28 March 1991)

\begin{abstract}
A method is given by which the response of a rotating or non-rotating Timoshenko beam can be determined, subjected to an accelerating fixed direction distributed surface force. The beam model includes the gyroscopically induced displacement transverse to the direction of the load. The solution for pinned supports is set up using multi-integral transforms, and the inversion is expressed in terms of convolution integrals. These are numerically integrated for a uniformly distributed load having an exponentially varying velocity function. Results are presented for the displacement under the load's center as a function of position and for the displacement of every point on the beam at an instant in time. Comparisons are made between the beam response to a constant velocity load and its response to a load which accelerates to the same velocity.
\end{abstract}

\section{INTRODUCTION}

This paper presents a study of the dynamic response of a rotating beam subjected to an axially distributed load acting normally to the top surface. The load has constant magnitude and accelerates axially along the beam's surface; the beam is pinned and rotates with constant angular velocity.

Studies in the area of constant velocity loads on non-rotating beams are extensive, see for example, references [1] and [2]. The response of a rotating beam subjected to a constant velocity point force has been studied in reference [3], including gyroscopic effects, and in reference [4], where gyroscopic effects have not been included, but the loading is deflection dependent. In reference [5], the resonance of a rotating cylindrical shell was studied for constant speed loads having harmonic magnitude; the effects of internal pressure and Coriolis acceleration were included.

An application of the class of problems involving moving loads on rotating structures is found in machining where the moving force simulates a tool and the structure a workpiece. The motivation given by the authors in references [3] and [4] was, in part, to study the dynamic effects that would arise in future, very high speed, machining processes (see reference [6]). In addition, the problem studied in reference [5] could be applied to a machining process in which the load magnitude is harmonic.

The present work is a generalization of the problem studied in reference [3]. Machining operations in general may involve varying conditions, including tool speed, as described in reference [7]. The present study could be applied to high speed machining problems 
having varying speed distributed loads. In addition, by putting the angular velocity to zero, the solution here can be applied to problems in which an accelerating distributed force passes over a beam, as for example in pressure wave problems.

The beam model used here is based on the Timoshenko theory. $\Lambda$ s described in reference [3], such a model includes the displacement component transverse to the load direction, which is gyroscopically induced by interaction between the displacement in the direction of the load and the beam rotation. The problem is set up in terms of a general load having fixed direction, and the solution is then expressed in terms of multi-integral transforms (Laplace and finite Fourier) for this general load. Only in the last step of the Laplace inversion process is a load type specified. Here, the inversion is carried out for a uniformly distributed load traveling with velocity exponentially approaching some asymptotic value. The final solution is set up in terms of a series of convolution integrals which are numerically integrated.

\section{EQUATIONS OF MOTION}

The beam geometry is shown in Figure 1; it has length $l$ and a circular cross-section of radius $a$. The $x, y$ and $z$ system is fixed in space at the centroid of the left end of the beam, and displacement components of the beam's centroidal axis in the $x$ and $y$ directions are denoted by $u_{1}$ and $u_{2}$, respectively. The beam rotates with constant angular speed $\Omega$ about the $z$-axis. A surface load $P(z, t)$ acts in the $x$ direction and moves in the $z$ direction. $d(t)$ denotes the location of the load's centroid as a function of time.

The linear equations of motion for a rotating Timoshenko circular beam subjected to a fixed direction force can be found in reference [3] and are given below:

$$
\begin{gathered}
\rho A \ddot{u}-K A G\left(u^{\prime \prime}-\psi^{\prime}\right)=P(z, t), \\
E I \psi^{\prime \prime}+K A G\left(u^{\prime}-\psi\right)-\rho I(\ddot{\psi}-2 \mathrm{i} \Omega \dot{\psi})=0,
\end{gathered}
$$

where $\rho$ is the material's density, $A$ is the cross-sectional area, $G$ is the shear modulus, $K$ is the Timoshenko shear coefficient, $E$ is the modulus of elasticity and $I$ is the moment of inertia of the circular cross-section. Here primes and dots denote differentiation with respect to $z$ and $t$ respectively; $u$ and $\psi$ are defined by

$$
u=u_{1}+\mathrm{i} u_{2}, \quad \psi=\psi_{1}+\mathrm{i} \psi_{2} .
$$

$\psi_{1}$ and $\psi_{2}$ are the portions of the centroidal axis' slope due to bending. Defining $\beta_{1}$ and $\beta_{2}$ as the portions of its slope due to shearing results in

$$
u_{1}^{\prime}=\psi_{1}+\beta_{1}, \quad u_{2}^{\prime}=\psi_{2}+\beta_{2} \text {. }
$$

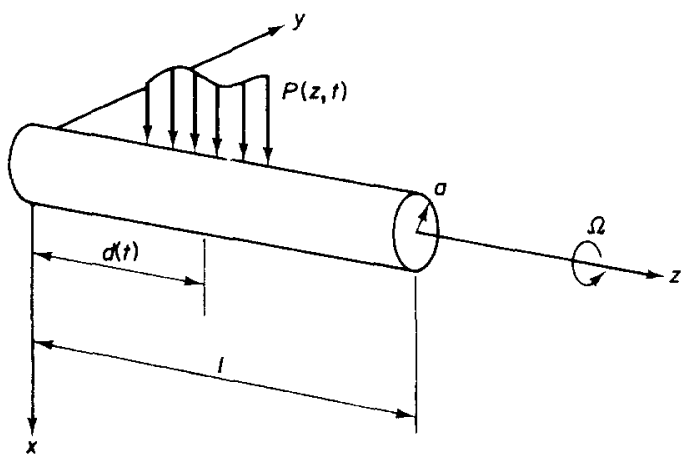

Figure 1. Beam geometry. 
Pinned boundary conditions are assumed at $z=0$ and $z=l$, which results in

$$
u=0, \quad z=0, l, \quad \psi^{\prime}=0, \quad z=0, l .
$$

Equations (1) and (2) neglect damping but include rotary inertia and gyroscopic moments which appear as the $\ddot{\psi}$ and $\dot{\psi}$ terms, respectively. The effect of the gyroscopic term is to induce a displacement component perpendicular to the direction of the load.

\section{SOLUTION OF THE EQUATIONS OF MOTION}

Equations (1) and (2) form a pair of coupled linear partial differential equations. Since the load moves with varying velocity, the non-homogeneity appearing in equation (1) is not of a simple form, thereby complicating determination of the particular part of the solution. Here the solution is obtained by multi-integral transforms. Finite Fourier transforms are used in space and the Laplace transform is used in time. Inversion of the Laplace transform is obtained by partial fraction expansion along with numerical integration of convolution integrals.

Applying the finite Fourier transform in space and the Laplace transform in time (zero initial conditions) to equations (1) and (2) gives

$$
\left(s^{2}+a_{1}\right) \tilde{\hat{u}}-a_{2} \tilde{\hat{\psi}}=F(s), \quad\left(s^{2}-2 \mathrm{i} \Omega s+a_{6}\right) \tilde{\hat{\psi}}-a_{4} \tilde{\hat{u}}=0,
$$

where the boundary conditions have been applied, and the finite Fourier transforms are defined by

$$
\hat{u}=\int_{0}^{l} u \sin \left(\frac{n \pi z}{l}\right) \mathrm{d} z, \quad \hat{\psi}=\int_{0}^{l} \psi \cos \left(\frac{n \pi z}{l}\right) \mathrm{d} z .
$$

The Laplace transform is denoted by a tilde $(\sim)$, and its parameter is $s$. Also,

$$
F(s)=\frac{1}{\rho A} \mathscr{L}\left\{\int_{0}^{l} P(z, t) \sin \left(\frac{n \pi z}{l}\right) \mathrm{d} z\right\}
$$

and

$$
\begin{gathered}
a_{1}=c_{2}^{2}(n \pi / l)^{2}, \quad a_{2}=c_{2}^{2}(n \pi / l), \\
a_{4}=K A G n \pi(\rho l l), \quad a_{6}=c_{1}^{2}(n \pi / l)^{2}+K A G /(\rho I), \\
c_{1}^{2}=E / \rho, \quad c_{2}^{2}=K G / \rho .
\end{gathered}
$$

Note that the boundary conditions (5) and (6) are satisfied by the inversion of the forms (9) and (10). Other types of boundary conditions will be discussed later.

The solutions of equations (7) and (8) are

$$
\tilde{\hat{\psi}}=a_{4} F(s) / \xi(s), \quad \tilde{\hat{u}}=\left(s^{2}-2 \mathrm{i} \Omega s+a_{6}\right) F(s) / \xi(s),
$$

where

$$
\begin{gathered}
\xi(s)=s^{4}-2 \mathrm{i} \Omega s^{3}+a_{8} s^{2}-2 \mathrm{i} \Omega a_{1} s+a_{7}, \\
a_{7}=c_{1}^{2} c_{2}^{2}(n \pi / l)^{4}, \quad a_{8}=a_{1}+a_{6} .
\end{gathered}
$$

Before the inverse transformations can be evaluated, a specific load form must be assumed. Here the load is taken to start with zero velocity at $z=0$ and accelerate to some 
steady speed $V_{0}$ according to the relation

$$
V=V_{0}\left(1-\mathrm{e}^{-\alpha t}\right)
$$

where $\alpha$ is a parameter controlling the rapidity with which the steady speed is attained.

The location of the load's centroid as a function of time is then given by

$$
d(t)=V_{0}\left(t+\frac{1}{\alpha}\left(\mathrm{e}^{-\alpha t}-1\right)\right) .
$$

A uniformly distributed load having width $2 q$ can then be expressed in the functional form:

$$
P(z, t)= \begin{cases}P_{1}^{0}, & |z-d(t)|<q \\ 0, & \text { otherwise }\end{cases}
$$

where $P_{1}^{0}$ is a constant.

Next, the inverse Laplace transformation of equation (13) is expressed by means of the convolution theorem, giving

$$
\hat{u}=P_{n} \int_{0}^{\prime} \zeta\left(t-t^{\prime}\right) \sin \left(n \pi d\left(t^{\prime}\right) / l\right) \mathrm{d} t^{\prime},
$$

where

$$
P_{n}=2 \sin (n \pi q / l) l P_{1}^{0} /(n \pi \rho A), \quad \zeta(t)=\mathscr{L}^{-1}\left\{\left(s^{2}-2 \mathrm{i} \Omega s+a_{6}\right) / \xi(s)\right\} .
$$

Note that the use of the convolution theorem permits the inversion to be expressed without calculation of the Laplace transformation (11).

$\zeta(t)$ is determined by partial fraction expansion; then equation (17) becomes

$$
\hat{u}=P_{n} \int_{0}^{t}\left(\sum_{j=1}^{4} A_{j} \mathrm{e}^{r_{j}\left(t-t^{\prime}\right)}\right) \sin \left(n \pi d\left(t^{\prime}\right) / l\right) \mathrm{d} t^{\prime},
$$

where the $r_{j}$ are the zeros of $\xi(s)$, and

$$
A_{j}=\frac{r_{j}^{2}-2 \mathrm{i} \Omega r_{j}+a_{6}}{4 r_{j}^{3}-6 \mathrm{i} \Omega r_{j}^{2}+2 a_{8} r_{j}-2 \mathrm{i} \Omega a_{1}} .
$$

On physical grounds it can be argued that the roots of $\xi(s)$ are purely imaginary and then equation (18) can be written in the form

$$
\hat{u}=P_{n}\left(-I_{1}+\mathrm{i} I_{2}\right)
$$

where

$$
\begin{aligned}
& I_{1}=-\int_{0}^{t}\left(\sum_{j=1}^{4} A_{j}^{I} \sin \left(r_{j}^{I}\left(t-t^{\prime}\right)\right)\right) \sin \left(n \pi d\left(t^{\prime}\right) / l\right) \mathrm{d} t^{\prime}, \\
& I_{2}=\int_{0}^{t}\left(\sum_{j=1}^{4} A_{j}^{I} \cos \left(r_{j}^{I}\left(t-t^{\prime}\right)\right)\right) \sin \left(n \pi d\left(t^{\prime}\right) / l\right) \mathrm{d} t^{\prime},
\end{aligned}
$$

The $r_{j}^{I}$ and the $A_{j}^{I}$ are the imaginary parts of $r_{j}$ and $A_{j}$, respectively. 
Inversion of the Fourier transform followed by separation into real and imaginary parts gives:

$$
\begin{aligned}
& \frac{u_{1}}{u_{0}}=-\frac{24 E a^{2}}{q l^{3} \rho \pi} \sum_{n=1}^{\infty} \frac{\sin (n \pi q / l)}{n} I_{1} \sin (n \pi z / l), \\
& \frac{u_{2}}{u_{0}}=\frac{24 E a^{2}}{q l^{3} \rho \pi} \sum_{n=1}^{\infty} \frac{\sin (n \pi q / l)}{n} I_{2} \sin (n \pi z / l),
\end{aligned}
$$

where $u_{0}=P_{1}^{0} q l^{3} /(24 E I)$.

Note that $u_{0}$ is the static deflection at $z=l / 2$ of the same beam subjected to a point force at $z=l / 2$ having magnitude $2 q P_{1}^{0}$.

Closed form evaluation of the integrals $I_{1}$ and $I_{2}$ is not possible because of the complicated nature of $d(t)$, which results from having a variable load speed. Instead, numerical integration for each $n$ at every time of interest is employed.

The solution for a non-rotating pinned Timoshenko beam subjected to the transient surface line load is extracted from the present solution by setting $\Omega=0$.

Although not pursued in depth here, it should be noted that a different solution procedure would be required for boundary conditions other than pinned-pinned, since use of finite Fourier transforms would not lead to their satisfaction. Modal analysis and Galerkin's method have been used in reference [8] to solve for the response of a rotating clampedpinned Rayleigh beam subjected to a constant speed load.

A possible approach in the present situation is to suppress the spatial dependence using Galerkin's method, with comparison functions appropriate to the problem at hand. The procedure would yield a set of coupled, non-homogeneous, constant coefficient ordinary differential equations. These could then be reduced to a set of algebraic equations in the Galerkin coefficients by means of the Laplace transformation. However, the set of equations is infinite, and so one would not obtain results as simple as equations (12) and (13). Truncation would lead to equations similar to equations (12) and (13), but with high degree polynomials in the numerator and denominator. At that point, whether inversion is by means of the convolution theorem, or by strictly numerical means, is left as an open question.

\section{NUMERICAL RESULTS}

In this section, numerical results are presented for rotating and non-rotating beams subjected to the varying speed surface load. Comparisons are made with results obtained by specialization to the constant speed load case $(\alpha \rightarrow \infty)$. In addition, results for this specialized case are compared to previously published ones.

The integrals $I_{1}$ and $I_{2}$, and the zeros of the complex polynomial $\xi(s)$, were numerically calculated using IMSL version 10 subroutines. In all instances, truncation of the series in equations (21) and (22) at 30 terms provided excellent convergence. The numerical computations were performed on the University of Michigan MTS system (IBM 3090); a typical computation of $u_{1}$ and $u_{2}$ at a single time and load location took approximately $6 c p u$ on this system.

In order to facilitate presentation of the graphical output, the following non-dimensional parameters will be referred to as in reference [3]:

$$
\tilde{R}=\pi a /(2 l), \quad \tilde{V}=V_{0} /\left(\tilde{R} c_{1}\right), \quad \tilde{\Omega}=\Omega / \omega_{1},
$$

where $\omega_{1}$ is the fundamental natural frequency of a non-rotating pinned Euler-Bernoulli beam. 
The following values were used for the parameters unless otherwise indicated: $E=$ $207 \times 10^{9} \mathrm{~N} / \mathrm{m}^{2}, \rho=7700 \mathrm{~kg} / \mathrm{m}^{3}, l=1 \mathrm{~m}, \tilde{R}=0.15, \tilde{\Omega}=2.5, K=0.9$. It should be noted that numerical parameter studies involving the load width $2 q$ have been conducted. Results of these studies (not given here) show that moderate variation of $q$ has an insignificant effect on the beam's response. Unless otherwise indicated, a value of $q=0.005 \mathrm{~m}$ has been used throughout (which corresponds to $l /(2 q)=100$ ).

The solution and numerical procedure developed here can be partially validated by comparison of results obtained by specialization of the present solution with results in reference [3]. By letting $q \rightarrow 0$ and $\alpha \rightarrow \infty$, the special case of a point load traveling with constant speed can be considered. Comparison of such results with results in Tables 2 and 3 of reference [3] are shown in Table 1. Very close agreement is seen throughout.

\section{TABLE 1}

Comparison with values from Tables 2 and 3 of reference [3] for a point load. V constant, $\widetilde{R}=0 \cdot 15, \tilde{\Omega}=2 \cdot 5$. Values shown are maximum displacements under the load

\begin{tabular}{cll}
\hline$\tilde{V}$ & Present study & Reference [3] \\
\hline 0.111 & $u_{1} / u_{0}=1 \cdot 130$ & $u_{1} / u_{0}=1.114$ \\
0.500 & $u_{1} / u_{0}=1.704$ & $u_{1} / u_{0}=1.704$ \\
1.100 & $u_{1} / u_{0}=0.943$ & $u_{1} / u_{0}=0.942$ \\
1.500 & $u_{1} / u_{0}=0.633$ & $u_{1} / u_{0}=0.634$ \\
0.500 & $u_{2} / u_{0}=0.1307$ & $u_{2} / u_{0}=0.1307$ \\
\hline
\end{tabular}

A typical plot of the load speed function given by equation (14) is shown in Figure 2. $V(t)$ exponentially approaches the steady value $V_{0}$. In all cases considered, $\alpha$ was chosen so that $V(t)$ reaches $0.99 V_{0}$ at approximately $0.3 l$. In Figure $2, \alpha=1000$ and $\tilde{V}=0.11$.

The displacements $u_{1}$ (there is no $u_{2}$ in this case) for a non-rotating beam are shown in Figure 3 for a constant speed load and a varying speed load, with $\tilde{V}=0 \cdot 219$. The curves show the displacement at the center point of the load as the load traverses the beam. The two curves differ significantly in the middle region but have the same overall general

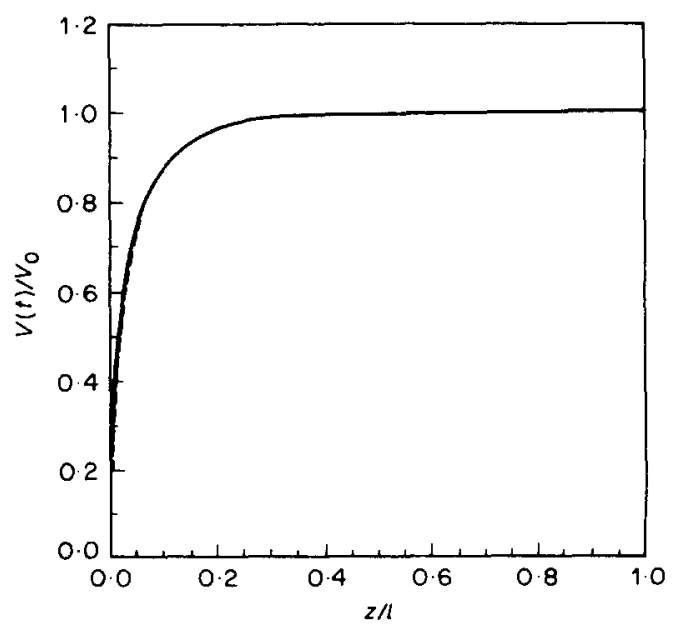

Figure 2. Load speed as a function of load position. 


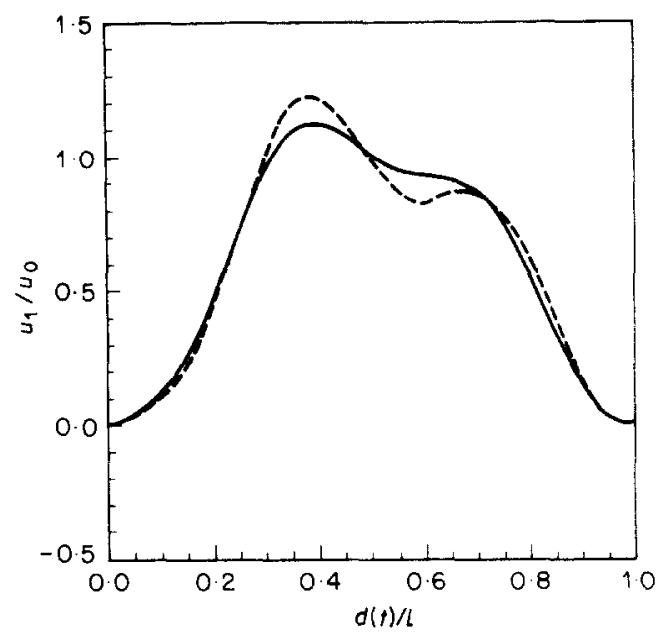

Figure 3. Displacement under load as a function of load position for a non-rotating beam : - $V(t) ;-\cdots$, $V=$ constant.

character. The maximum difference between the two curves occurs at $d(t) / l=0 \cdot 6$, where the displacement for the varying speed load is $12 \%$ greater than the displacement for the constant speed load (relative to the constant speed case). At $d(t) / l=0 \cdot 38$, the location of both maxima, the displacement for the varying speed load is $7 \%$ less than its counterpart.

All subsequent results are for rotating beams. The displacements $u_{1}$ and $u_{2}$ under the load center as functions of load position are plotted in Figures 4 and 5 for both the constant and varying speed load cases at $\vec{V}=0 \cdot 11$. In Figure 4 , the two curves for $u_{1}$ differ (at $d(t) / l=0.54$ ) by at most $7 \%$, with the variable speed case giving the larger value. The difference between the peak values is about $6 \%$ with the constant speed case giving the larger value. Note that the peak for the varying speed case lags the peak for the constant speed case.

The varying speed load is seen to have a more pronounced effect on the gyroscopically induced displacement, $u_{2}$ (see Figure 5), with the peak values now differing by about $50 \%$. Again the constant speed case gives the larger peak value.

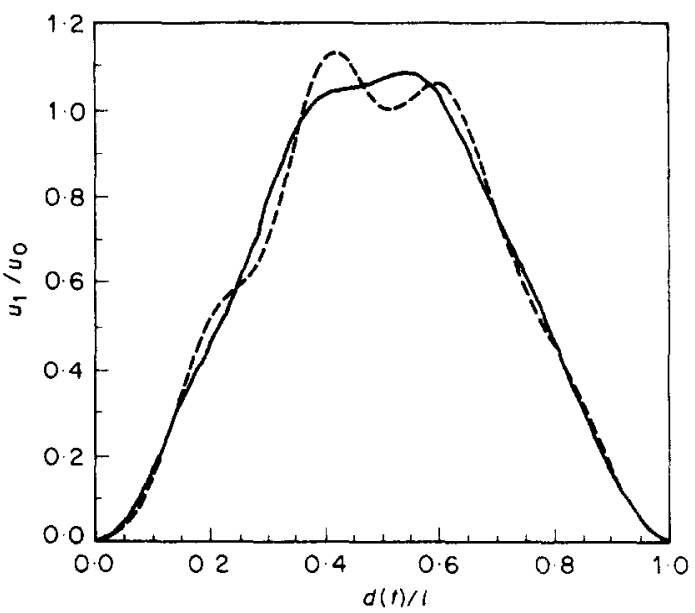

Figure 4. Displacement $u_{1}$ under load center as a function of load position:,$- V(t) ;-\cdots, V=$ constant. 


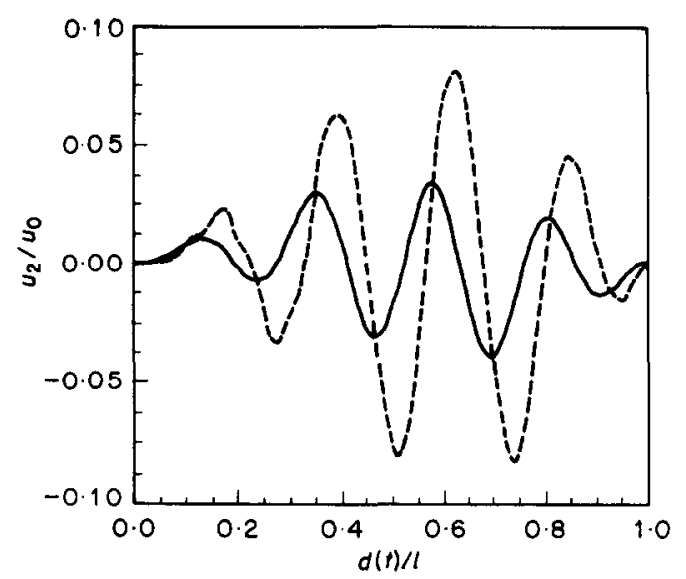

Figure 5. Displacement $u_{2}$ under load center as a function of load position: -,$- V(t) ;-\cdots, V=$ constant.

Additional results are given in Figures 6 and 7. In Figure 6 is given the percentage difference of the overall maximum $u_{1}$ under the load for varying $V$ with respect to that for constant $V$ as a function of $\tilde{V}$. The analogous plot for $u_{2}$ is shown in Figure 7. (For fixed $\tilde{R}$, changing $\tilde{V}$ may be thought of as varying the asymptotic load speed.) Both figures exhibit a significant dependence on $\tilde{V}$. The maximum difference between the $u_{1}$ values is about $7 \%$ and arises at $\tilde{V}=0 \cdot 219$. For the parameters used here, this corresponds to a value of $V_{0}=170 \mathrm{~m} / \mathrm{s}$, which is in the range of speeds reported for "very high speed machining", as described in reference [6].

For $u_{2}$ (see Figure 7), the effect of the varying speed is seen to be very pronounced for slower speeds, but rapidly decreases, becoming insignificant for speeds in the "ultra high" or ballistic machining range $(\tilde{V}>0 \cdot 32$, i.e., $V>250 \mathrm{~m} / \mathrm{s})$.

It should be emphasized that Figures 6 and 7 give the percentage differences in the maximum displacement of the beam at the point corresponding with the center of the load. Frequently, the maxima for the two cases do not occur at the same value of $d(t)$ (see Figure 4, for example), and there often is a significant percent difference between

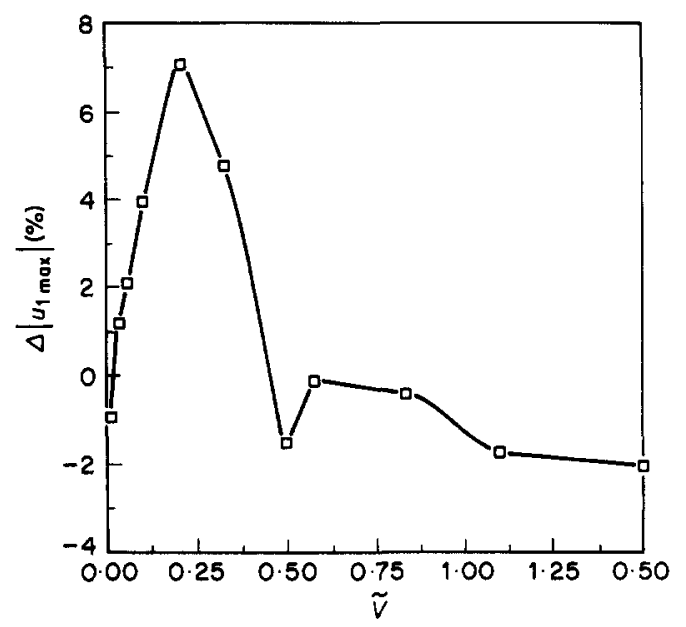

Figure 6. Percentage difference between peak values of $u_{1}$ under load as a function of load speed. 


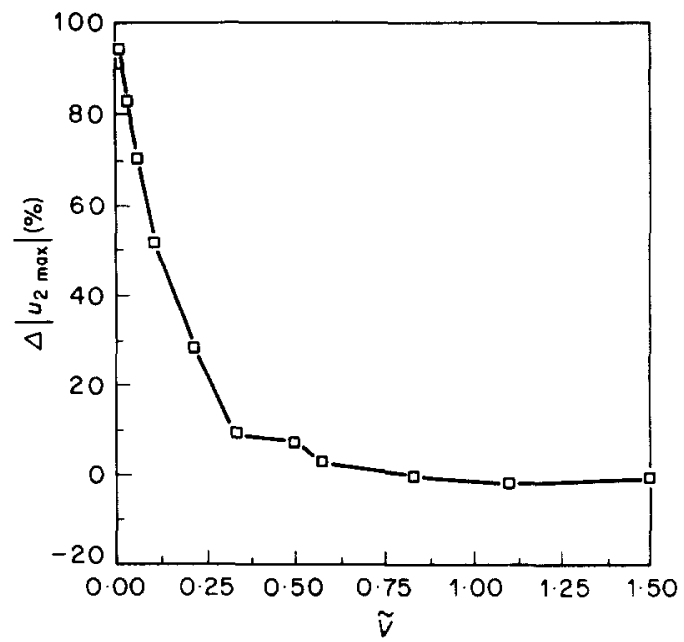

Figure 7. Percentage difference between peak values of $u_{2}$ under load as a function of load speed.

displacement pairs at a value of $d(t)$ which does not correspond to the maximum displacement for either case (see Figure 3, for example).

Lastly, in Figures 8 and 9 is shown the response of every point on the beam for $\tilde{V}=$ 0.11 , when the load is at $d(t) / l=0.026$. These figures give "snapshots" of the beam's response. Again, the varying speed case and the constant speed case are given together in each figure. Note, the load reaches the given location at two different times for the two cases, so that the two curves in each figure correspond to two different times. The figures reveal that the maximum values of $u_{1}$ and $u_{2}$ at the given instant do not correspond to the point under the load's center. Instead, the maximum value of each displacement component occurs at a point ahead of the load. However, in most cases, by the time the load reaches about $d(t) / l=0 \cdot 3$, the maximum displacement usually occurs at the load's center. The figures also show that the varying speed case has significantly larger displacements than the constant speed case. This is because the varying speed curve corresponds to a later time than the constant speed curve. Therefore, the response for the varying speed case has

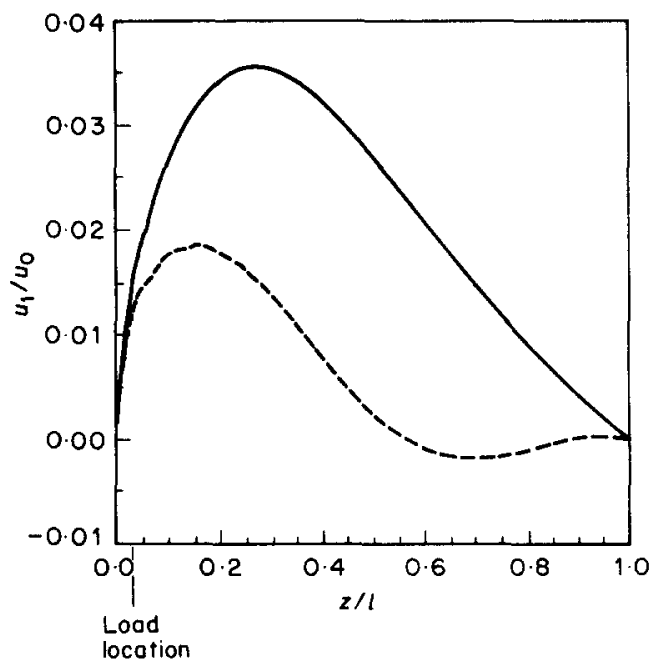

Figure 8. Instantaneous displacement $u_{1}$ as a function of $z:-, V(t) ;---, V=$ constant. 


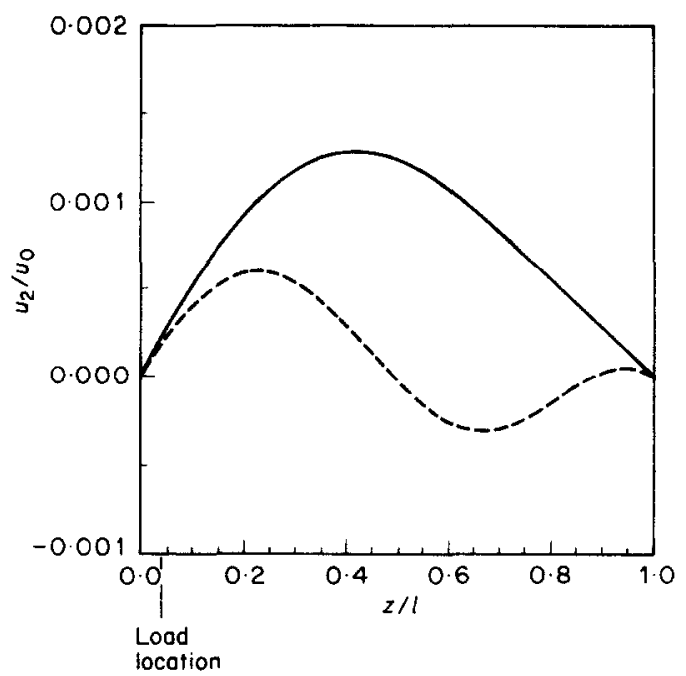

Figure 9. Instantaneous displacement $u_{2}$ as a function of $z:-, V(t) ;--, V=$ constant.

had more time to build than that for the constant speed case. For later load locations, the large difference between the two curves tends to moderate.

\section{CONCLUSIONS AND DISCUSSION}

A general method has been developed to treat an accelerating fixed direction distributed surface force on a rotating, pinned Timoshenko beam. The analysis includes the gyroscopically induced displacement component transverse to the load direction.

Results are presented for beams subjected to a uniformly distributed surface load having an exponential velocity function. These results show that the effect of varing speed load on the beam's maximum displacement under the load is highly dependent on the asymptotic speed being approached by the load. In general, the transverse displacement has been found to be effected more than the displacement in the direction of the load. For the range of speeds considered, the percentage differences between the peak values under the load vary from 0 to $90 \%$ for $u_{2}$, and from $-2 \%$ to $7 \%$ for $u_{1}$. The varying speed load function used here usually leads to smaller overall peak displacements under the load. This leads one to conjecture that improvements in machining accuracy could be achieved through use of variable speeds. Since the gyroscopically induced component, $u_{2}$, is significantly effected, accuracy in processes involving high workpiece rotational speed can be particularly improved.

Results giving "snapshots" of the beam's response for early load locations show significant difference between the two cases, and that the maximum displacement is not necessarily under the load. For machining, primary interest is in the response at points under the load (the tool) since this effects accuracy. However, for other problems in which a force rapidly passes over a beam-like structure, the response of the whole structure, at every instant, should be studied.

Some directions for possible future research are now outlined. The solution approach presented here can be used to determine the response to other load velocity functions and distributions, provided the load direction is fixed and the beam is pinned. Varying load magnitude, as might arise in machining with multi-point cutters, high speed cylindrical grinding or situations in which cutting overlap is present, can also be treated using the 
present approach. Of particular interest is periodic load magnitude, which could lead to dynamic instability. The effects of various boundary conditions also could be studied. As described, this would require a modified solution approach. Also, a common model of cutting involves deflection dependent forces (see reference [4]). Such a model, together with non-constant load velocity, warrants study but requires the development of a new solution methodology, since time dependent coefficients appear in the differential equations of motion.

\section{REFERENCES}

1. C. R. Steele 1967 Journal of Applied Mechanics 34, 111-118. The finite beam with a moving load.

2. L. FRYBA 1972 Vibration of Solids and Structures Under Moving Loads. Gronigen, The Netherlands: Noordhoff.

3. R. Katz, C. W. Lee, A. G. Ulsoy and R. A. Scott 1988 Journal of Sound and Vibration 122, 131-148. The dynamic response of a rotating shaft subject to a moving load.

4. R. KAtZ, C. W. LEE, A. G. Ulsoy and R. A. Scott 1987 Journal of Vibration, Acoustics, Stress, and Reliability in Design 109, 361-365. Dynamic stability and response of a heam subject to a deflection dependent moving load.

5. S. C. HuANG and B. S. Hsu 1990 Journal of Sound and Vibration 136, 215-228. Resonant phenomena of a rotating cylindrical shell subjected to a harmonic moving load.

6. D. G. Flom, R. Komanduri and M. Lee 1984 Anmual Review of Material Science 14, 231-278. High-speed machining of metals.

7. B. K. Fussell and K. SRINIVASAN 1989 Journal of Engincering for Industry 111, 27-36. An investigation of the end milling process under varying machining conditions.

8. C. W. Lee, R. Katz, A. G. Ulsov and R. A. Scott 1988 Journal of Sound and Vibration 122. 119-130. Modal Analysis of a distributed parameter rotating shaft. 